\title{
Desenvolvimento da versão em português do Brasil do questionário sobre padrão de relacionamentos Beziehungs-Muster Fragebogen
}

\author{
Eliane Bernadete Ferreira* \\ Maria Lúcia Tiellet Nunes** \\ Regina A. Kurth*** \\ Dan Pokorny**** \\ Luciana Terra\# \\ Simone Hauck\#\# \\ Lúcia Helena Freitas Ceitlin\#\#\#
}

\section{INTRODUÇÃO}

O Beziehungs-Muster Fragebogen (BeMus-3), conhecido na literatura internacional como Relationship Patterns Questionnaire $(\mathrm{RPQ})^{1,2}$, é um questionário que

Estudo realizado no Programa de Psicoterapia Psicanalítica, Hospital de Clínicas de Porto Alegre (HCPA), Porto Alegre, RS. Baseado em trabalho de dissertação de mestrado da primeira autora, intitulado "Um estudo da relação entre o padrão transferencial e a aliança terapêutica de pacientes em psicoterapia psicanalítica", apresentado à Universidade Federal do Rio Grande do Sul em 2006

* Psiquiatra. Mestranda, Programa de Pós-Graduação em Ciências Médicas: Psiquiatria, Universidade Federal do Rio Grande do Sul (UFRGS), Porto Alegre, RS.

** Psicóloga. PhD. Professora titular, Curso de Psicologia, Pontifícia Universidade Católica do Rio Grande do Sul (PUCRS), Porto Alegre, RS Coordenadora, Programa de Pós-Graduação em Psicologia, PUCRS, Porto Alegre, RS.

*** Universidade de Giessen, Alemanha.

**** Universidade de Ulm, Alemanha.

\# Psicóloga. Mestranda, Programa de Pós-Graduação em Ciências Médicas: Psiquiatria, UFRGS, Porto Alegre, RS.

\#\# Psiquiatra. Doutoranda, Programa de Pós-Graduação em Ciências Médicas: Psiquiatria, UFRGS, Porto Alegre, RS.

\#\#\# Psiquiatra. MpH, PhD. Professora adjunta, Departamento de Psiquiatria e Medicina Legal, Faculdade de Medicina, UFRGS, Porto Alegre, RS avalia o padrão central de relação, podendo, então, ser usado para avaliação da transferência. $O$ construto transferência teve sua importância reconhecida desde que Sigmund Freud lançou sua teoria em $1905^{3}$, no pós-escrito do "caso Dora". O autor definiu transferência como novas edições ou facsímiles de impulsos e fantasias que são despertados e tornados conscientes durante o progresso da análise. Experiências psicológicas são revividas, mas não mais dirigidas às figuras primitivas do passado, e, sim, à figura do médico na situação atual.

Freud $^{3}$ colocou a transferência como um obstáculo predestinado à psicanálise que, se for detectada e interpretada ao paciente, tornase seu mais poderoso aliado. Em Cinco lições de psicanálise ${ }^{4}$, Freud teorizou o processo da transferência. Expelido da consciência e da lembrança, o impulso continua a existir no inconsciente, à espera de oportunidade para se revelar, havendo a formação de um substituto do reprimido, para lançar à consciência algo que também traz a sensação de desprazer que se julgava resolvida pela repressão. 
Melanie Klein ${ }^{5}$ também trouxe contribuições importantes para esse construto. Atribuiu a origem da transferência a um momento anterior ao sugerido por Freud. Situou a transferência como oriunda das relações primitivas de objeto, das primeiras relações da criança com o seio.

Freud $^{6}$ afirmou que cada indivíduo desenvolve uma forma própria de conduzir-se. Essa forma se dá através da ação combinada de sua disposição inata e das influências sofridas nos primeiros anos e apresenta-se como um clichê estereotípico repetido no decorrer da vida.

Outros autores continuam trazendo contribuições a esse tema, que nunca deixou de ser atual, pois, se é instrumento de cura, como disse seu descobridor, é instrumento diário de trabalho. Sua compreensão, então, pode nos aproximar do paciente e, possivelmente, trazer um melhor prognóstico ao tratamento.

Só na base de dados PsyclNFO, encontramos 12.125 referências sobre estudos acerca do tema, desde 1971 até junho de 2006. Se usarmos a palavra transference como descritor maior, encontraremos 3.552 estudos nessa base de dados, sendo 1.073 estudos empíricos, 39 revisões de literatura, 10 estudos qualitativos, oito estudos quantitativos e demais com outras metodologias. A natureza dos trabalhos estende-se desde abordagens puramente psicanalíticas até associações entre o construto e transtornos psiquiátricos.

Nesse contexto, vem crescendo o número de instrumentos disponíveis para aferição do padrão central de relacionamentos ou dos clichês que se repetem no decorrer da vida. Podemos classificar esses instrumentos em observacionais e auto-aplicáveis.

Dentre os instrumentos observacionais, podemos citar o Core Conflictual Relationship Theme $(\mathrm{CCRT})^{7}$, o Plan Formulation Method ${ }^{8}$, o Configurational Analysis Method ${ }^{9}$, o Frame Method $^{10}$, o Cyclical Maladaptive Pattern Method $^{11}$, o Idiographic Conflict Formulation Method $^{12}$ e o Consensual Response Formulation Method ${ }^{13}$.

Entre as medidas auto-aplicáveis, citamos - INTREX Questionnaire ${ }^{14}$, o Central Relationship Questionnaire ${ }^{15}$ e o BeMus- $3^{1,2}$.

Existem instrumentos robustos, tanto observacionais quanto auto-aplicáveis, como os citados nos dois parágrafos anteriores. Sem querer traçar um comparativo entre as duas formas de avaliar a transferência, pois isso seria bastante complexo, citamos algumas vantagens dos instrumentos auto-aplicáveis. De maneira geral, são instrumentos de baixo custo de aplicação e análise, pois dispensam um número maior de pessoas envolvidas para sua avaliação (juízes), e são de aplicação mais rápida e mais objetivos.

O BeMus-3 é um questionário autoaplicável baseado no modelo interpessoal de Benjamin $^{16}$ e na CCRT ${ }^{7}$.

Neste estudo, relatamos o desenvolvimento da versão em português do Brasil desse instrumento, em sua forma abreviada, para uso em estudos exploratórios de investigação sobre o construto transferência, alguns dos quais, neste momento, já foram realizados por nossa equipe de pesquisa ou estão em fase de conclusão. O objetivo principal seria, então, a introdução, em nosso meio, de um instrumento para avaliação da transferência que tenha um bom suporte teórico e seja de aplicação compatível com nossas condições socioeconômicas.

\section{DESCRIÇÃO DO INSTRUMENTO}

O BeMus-3 $3^{1,2}$, RPQ na literatura internacional, é um questionário desenvolvido pelos pesquisadores Regina Kurth e Dan Pokorny, validado em $2002^{1}$ e $2004^{2}$. Apresenta uma versão longa e outra abreviada. O desenvolvimento da versão em português do Brasil, neste estudo, foi realizado para a versão abreviada.

É um instrumento auto-aplicável e pode ser aplicado a qualquer pessoa de referência e a períodos de tempo diferentes (presente ou infância).

Os itens e escalas do BeMus-3 têm sua teoria baseada no modelo interpessoal de Benjamin ${ }^{16}$, que desenvolveu o Structural Analysis of Social Behavior (SASB), com três focos: o transitivo, o intransitivo e o introjetivo. $\mathrm{O}$ transitivo é o eixo direcionado ao outro. $\mathrm{O}$ intransitivo é direcionado ao próprio sujeito. $\mathrm{O}$ introjetivo representa os processos intrapsíquicos.

O modelo SASB é detalhado, conceitualmente rigoroso e psicometricamente avançado, descendente do modelo original de Leary ${ }^{17}$. O SASB permite que comportamentos interpessoais ou seus análogos intrapsíquicos sejam representados matematicamente como pontos em uma das três coordenadas interrelacionadas de duas dimensões ${ }^{18}$. Qualquer processo interpessoal, então, pode ser descrito matematicamente, localizando-se o mesmo em um ou mais pontos do SASB. Similarmente, diferenças e mudanças podem também ser 
quantificadas através das propriedades matemáticas desse modelo ${ }^{18}$.

A versão do BeMus-3 usada neste estudo corresponde a um desenvolvimento posterior do BeMus-3 de oito escalas unipolares: 1) Imposição; 2) Aceitação; 3) Amor; 4) Ajuda; 5) Submissão; 6) Defesa; 7) Ataque; 8) Indiferença. A versão abreviada, utilizada neste estudo, apresenta quatro escalas unipolares: 1) Imposição; 2) Amor; 3) Submissão; 4) Ataque. De acordo com o modelo de Benjamin ${ }^{16}$, essas quatro escalas distribuem-se num espaço bidimensional com os eixos de afiliação (amor e ataque) e interdependência (imposição e submissão).

Assim, o BeMus-3 fornece um escore para cada escala, em três níveis diferentes: resposta do self (RS), resposta do objeto (RO) e introjetivo. O nível self avalia a resposta do sujeito frente a determinado comportamento de outra pessoa (terapeuta, amigo, filho, mãe, pai, etc.); o nível objeto avalia a crença do sujeito acerca do comportamento da outra pessoa, em resposta a determinado comportamento seu; e o nível introjetivo, o comportamento do sujeito após um confronto com a outra pessoa. O questionário, em sua forma abreviada, está, então, estruturado da seguinte maneira: 16 itens, divididos em quatro questões, avaliam a RS; os 16 itens seguintes avaliam a RO; e oito itens, o nível introjetivo. Cada item é mensurado através de escala Likert de 5 pontos. O escore é obtido pela média dos itens relacionados à respectiva escala, e, por fim, a média é subtraída de um valor de medida individual.

O BeMus-3 investiga, então, o padrão de relação baseado na resposta do indivíduo à conduta de outra pessoa (RS), no que o indivíduo imagina ser a resposta de outra pessoa à sua conduta (RO), e como esse indivíduo age após o confronto com essa pessoa (nível introjetivo).

Até junho de 2006, havia cinco publicações a respeito desse instrumento na base de dados PsycINFO, cujos enfoques eram: validação do instrumento'; introdução a duas diferentes aplicações do instrumento ${ }^{19}$; propriedades psicométricas do instrumento²; uma abordagem quantitativa de auto-avaliação de padrões interpessoais ${ }^{20}$; estudo de validação com uma amostra clínica ${ }^{21}$. A validade do construto foi realizada através da checagem da estrutura cíclica das escalas e da ordem cíclica de seus itens. As escalas foram, então, correlacionadas umas com as outras. A força de correlação foi dependente da posição no modelo interpessoal. fortemente, de forma positiva, umas com as outras; escalas mais distantes mostraram correlações positivas mais fracas ou correlações negativas; e escalas em pólos opostos atingiram o valor negativo máximo. Quanto à ordem cíclica dos itens, os coeficientes de concordância para os níveis self e objeto de todos os itens foram satisfatórios. A confiabilidade teste/reteste das escalas de sujeito e objeto também foi satisfatória.

Esse instrumento foi cedido para uso neste estudo pelos autores Regina Kurth \& Dan Pokorny, através de um trabalho de cooperação com os mesmos. Seu perfil psicométrico, na versão original em alemão, descrito no parágrafo anterior, sugeriu um instrumento confiável, apto a trazer resultados fidedignos sobre padrões de relacionamento transferencial.

\section{MÉTODO}

As etapas do desenvolvimento da versão em português do Brasil do BeMus-3 incluíram o modelo proposto por Reichenheim et al. ${ }^{22}$ para obtenção da equivalência semântica, que consiste de: tradução, retrotradução, apreciação formal de equivalência e interlocução com a população-alvo.

\section{Obtenção da licença dos autores}

O contato com a primeira autora, Regina Kurth, ocorreu, inicialmente, em novembro de 2004, após a publicação do estudo sobre as propriedades psicométricas do BeMus-3. Foram feitos sucessivos contatos via e-mail, através dos quais ficou combinado um trabalho de cooperação usando esse instrumento. Foram obtidos o instrumento em sua íntegra, nas versões longa e abreviada, e também licença para a tradução do mesmo.

\section{Tradução}

Foi realizada a partir do material em alemão, por tradutor oficial, bilíngüe, fluente em alemão e português. O material obtido foi revisado por profissional da área da psicologia, com fluência no idioma alemão, que realizou, junto com profissional da psiquiatria, os ajustes considerados adequados para a adaptação.

\section{Retrotradução}

Um tradutor alemão profissional, com fluência no idioma português, realizou essa etapa, sem tomar conhecimento do material 
original em alemão, traduzido para o português pelo tradutor citado no item II da metodologia.

\section{Julgamento da equivalência semântica}

Foi realizado pela autora do instrumento, Regina Kurth, que fez sugestões na versão retrotraduzida abreviada, num total de sete. A equipe trabalhou os ajustes lingüísticos para a versão em português do Brasil. Estes foram novamente traduzidos para o alemão e reenviados aos autores, até que fosse confirmada pelos mesmos a equivalência semântica do texto.

\section{Consenso acerca do desenvolvimento da versão em português}

Foi realizado por 12 profissionais da área da psiquiatria e da psicologia. Solicitou-se, aos profissionais, que relacionassem o conteúdo de cada item a uma escala do modelo interpessoal, avaliando-se aspectos da validade de conteúdo.

\section{Interlocução com a população-alvo}

A versão do instrumento foi apresentada, então, a 10 indivíduos de várias idades (entre 18 e 60 anos) e diversos níveis educacionais (fundamental, médio, superior), os quais preencheram a escala e expressaram sua opinião sobre a compreensão da mesma, avaliando-se aspectos da validade aparente.

\section{RESULTADOS}

Através das etapas descritas na metodologia, obteve-se uma versão para o português do Brasil do instrumento BeMus-3, usado para avaliação do padrão central da relação ou padrão transferencial.

Após a etapa de retrotradução, no julgamento da equivalência semântica, os autores do instrumento sugeriram alteração em sete palavras do questionário. Os ajustes lingüísticos foram feitos pela equipe de pesquisa com a supervisão de um tradutor profissional, fluente em alemão e português.

$\mathrm{Na}$ etapa de consenso acerca do desenvolvimento da versão em português do BeMus-3, realizada por 12 profissionais da área da psiquiatria e psicologia, avaliando a adequada correlação entre cada item e a escala correspondente, houve concordância de 100\% nessa correlação, coerente com a proposta do instrumento, ficando sugerida, então, a validade de conteúdo.

Na etapa de interlocução com a populaçãoalvo, os 10 pacientes que responderam ao questionário não apresentaram dificuldades no seu preenchimento, considerando o material compreensível. Através de julgamento subjetivo sobre o sentido de cada item, foram averiguados aspectos da validade aparente. Não foi feito, então, nenhum ajuste após essa última etapa da metodologia.

A versão final do BeMus-3 em português do Brasil teve também a forma do instrumento original mantida (anexo 1).

\section{DISCUSSÃO}

A transferência, construto-base na pesquisa de tratamentos na linha psicanalítica, já foi definida por Freud, em $1905^{3}$, como agente de resistência e cura. Através de seu entendimento, o terapeuta pode chegar à compreensão dos impulsos e fantasias do paciente dirigidos às figuras primárias e, conseqüentemente, do padrão de relacionamento usado pelo paciente.

Hentschel ${ }^{23}$ explica que o pequeno número de estudos exploratórios, de metodologia empírica, sobre aspectos da relação entre paciente e terapeuta deve-se, entre outros fatores, à descrença dos clínicos acerca da capacidade dos instrumentos disponíveis de avaliar a relação terapêutica. Infere, porém, que já se percebem mudanças nesse panorama, devido ao aumento das escalas bem fundamentadas teoricamente.

Nas últimas três décadas, principalmente, foi desenvolvida uma série de instrumentos para quantificar a relação terapêutica, ou seja, para traduzir essa compreensão em dados numéricos, passíveis de serem avaliados e de fornecerem resultados estatisticamente aceitos. Muitos desses métodos, porém, requerem vários profissionais envolvidos, necessitando, assim, de maior período de tempo para aplicação e análise, e, às vezes, são necessários vídeo ou gravador na sessão.

Nos últimos anos, medidas auto-aplicáveis vêm sendo desenvolvidas para suprir algumas dificuldades enfrentadas com os métodos observacionais. Porém, é importante ressaltar que esses comentários não visam comparar os diferentes métodos quanto à sua capacidade de medir as variáveis a que se propõem, visto que existem métodos observacionais já consagrados na literatura, com excelente referencial teórico. Objetivam, 
apenas, justificar a introdução de novos instrumentos que podem, talvez, adaptar-se bem aos nossos objetivos.

O BeMus-3 é, então, um questionário autoaplicável, de baixo custo de aplicação, que dispensa o uso de vídeos ou gravadores na sessão. A implementação de estudos usando esse instrumento vai responder mais profundamente acerca de sua real adaptabilidade e funcionalidade para avaliar a transferência em nosso meio.

Uma observação importante acerca do referido instrumento é de que o mesmo ainda se encontra em evolução no seu país de origem e é passível de sofrer novos ajustes nos próximos anos.

A introdução de instrumentos que avaliem construtos fundamentais como a transferência serve de suporte à pesquisa, podendo nos fornecer resultados que melhorem a qualidade dos tratamentos psicoterápicos.

\section{CONCLUSÕES}

Transferência é um construto fundamental para a pesquisa na linha psicanalítica e mesmo em outras áreas da psiquiatria. Vem aumentando, na literatura internacional, o número de estudos que investigam a relação entre transferência e transtornos psiquiátricos ou entre transferência e apego, por exemplo. A introdução, em nosso meio, de instrumentos desenvolvidos para avaliação do padrão transferencial poderá propiciar suporte para melhorar o entendimento de nosso paciente, visto que o tema envolve grande parte de seus processos mentais. BeMus-3 é um instrumento auto-aplicável, de rápida administração e análise. Seu custo é baixo, e apresenta um bom suporte teórico.

Ao se trabalhar com instrumentos de pesquisa, deve-se conhecer suas vantagens, aplicabilidades e também suas limitações. Cada situação de pesquisa é um contexto em particular, e generalizações podem trazer prejuízos ao estudo; ou seja, saber o que se deseja investigar e buscar a escala de medida que melhor cumpra os objetivos são passos decisivos para a obtenção de resultados confiáveis.

A presente versão do BeMus-3 foi realizada e considerada adequada por profissionais da área da psiquiatria e psicologia e compreensível pelos pacientes da amostra retirada da população-alvo. Fica disponível, então, uma versão do BeMus-3 desenvolvida para o português do Brasil, observados os aspectos de equivalência conceitual, itens e semântica.

\section{REFERÊNCIAS}

1. Kurth RA, Pokorny D, Körner A, Geyer M. The relationship patterns questionnaire (RPQ): validation by using a representative sample. Psychother Psychosom Med Psychol. 2002;52(3-4):179-88.

2. Kurth RA, Korner A, Geyer M, Pokorny D. Relationship patterns questionnaire (RPQ): psychometric properties and clinical applications. Psychother Res. 2004;14(4):418-34.

3. Freud S. Fragmento da análise de um caso de histeria (1905). In: Freud S. Edição standard brasileira das obras psicológicas completas de Sigmund Freud. 2.ed. Rio de Janeiro: Imago; 1987. v. 7. p. 12-117.

4. Freud S. Cinco lições de psicanálise: Leonardo da Vinci e outros trabalhos (1909). In: Freud S. Edição standard brasileira das obras psicológicas completas de Sigmund Freud. Rio de Janeiro: Imago; 1969. v. 11. p. 13-51.

5. Klein $\mathrm{M}$. The origin of transference. Int $\mathrm{J}$ Psychoanal. 1952;33:433-8.

6. Freud S. A dinâmica da transferência (1912). In: Freud S. Edição standard brasileira das obras psicológicas completas de Sigmund Freud. Rio de Janeiro: Imago; 1969. v. 12. p. 133-43.

7. Luborsky L. Measuring a pervasive psychic structure in psychotherapy: the Core Conflictual Relationship Theme. In: Freedman N, Grand S, eds. Communicative structures and psychic structures. New York: Plenum; 1977. p. 36795.

8. Weiss J. Theory and clinical observations. In: Weiss J, Sampson H, Mount Zion Psychotherapy Research Group. The psychoanalytic process: theory, clinical observations, and empirical research. J. Weiss $\mathrm{H}$, eds. New York: Guilford Press; 1986. p. 3-138.

9. Horowitz MJ. States of mind: analysis of change in psychotherapy. New York: Plenum; 1979.

10. Dahl H. Frames of mind. In: Dahl H, Kächele $H$, Thomä $H$, eds. Psychoanalytic process research strategies. New York: Springer; 1988. p. 51-65.

11. Schacht TE, Binder JL, Strupp HH. The dynamic focus. In: Strupp HH, Binder JL. Psychotherapy in a new key: a guide to time limited dynamic psychotherapy. New York: Basic Books; 1984. p. 65-109.

12. Perry JC, Luborsky L, Silberschatz G, Popp C. An examination of three methods of psychodynamic formulation based on the same videotaped interview. Psychiatry. 1989;52(3):302-23.

13. Horowitz MJ. Relationship schema formulation: role relationship models and intrapsychic conflict. Psychiatry. 1989;52(3):260-74.

14. Benjamin LS. Principles of prediction using structural analysis of social behavior. In: Zucker RA, Aronoff $\mathrm{J}$, Rabin AJ, eds. Personality and the prediction of behavior. New York: Academic Press; 1984. p. 121-74.

15. Barber JP, Foltz C, Weinryb RM. The Central Relationship Questionnaire: initial report. J Couns Psychol. 1998;45(2):131-42.

16. Benjamin LS. Structural analysis of social behavior. Psychol Rev. 1974;81:392-425.

17. Leary T. Interpersonal diagnosis of personality. New York: Ronald Press; 1957.

18. Dahl H, Kächele $H$, Thomä $H$, eds. Psychoanalytic process research strategies. Berlin: Springer-Verlag; 1988.

19. Pokorny D. Multiple perspectives: psychometrics beyond right and wrong: an introduction to two different aplications of the relationship questionnaire. Psychother Res. 2004;14(4):415-7.

20. Korner A, Drapeau M, Perry JC, Kurth RA, Pokorny D, Geyer M. Self-assessment of interpersonal schemas using 
the relationship patterns questionnaire: a quantitative approach. Psychother Res. 2004;14(4):435-52.

21. Kurth RA, Pokorny D. Der Beziehungs-MusterFragebogen (BeMus): Validierung anhand einer klinischen stichprobe. Psychother Psychosom Med Psychol. 2005;55(12):502-11.

22. Reichenheim ME, Moraes $\mathrm{CL}$, Hasselmann $\mathrm{MH}$. Equivalência semântica da versão em português do instrumento Abuse Assessment Screen para rastrear a violência contra a mulher grávida. Rev Saude Publica. 2000;34(6):610-6.

23. Hentschel U, Kiessling M, Rudolf G. Therapeutic alliance and transference: an exploratory study of their empirical relationship. J Nerv Ment Dis. 1997;185(4):254-62.

\section{RESUMO}

Introdução: $O$ desenvolvimento da versão em português do Brasil do Beziehungs-Muster Fragebogen, conhecido na literatura internacional como Relationship Patterns Questionnaire, questionário que avalia o padrão central de relação, visa à introdução, em nosso meio, de uma medida auto-aplicável para implementação de projetos de pesquisa cuja questão envolva o construto transferência.

Metodologia: As etapas para o desenvolvimento da versão foram: obtenção da licença dos autores; tradução do instrumento original para o português do Brasil; julgamento e ajuste do material traduzido por profissionais da área da psiquiatria e psicologia; retrotradução; julgamento da equivalência semântica; consenso de profissionais da área da psiquiatria $e$ psicologia sobre a adequação do instrumento à nossa cultura; interlocução com a população-alvo.

Conclusão: $O$ Beziehungs-Muster Fragebogen é uma medida auto-aplicável, para avaliação da transferência ou padrão central da relação, que poderá facilitar a implementação de projetos para investigar os aspectos da relação terapêutica adjuntos à transferência. É um método de fácil aplicação e análise, baixo custo e que dispensa o uso de vídeos ou gravadores na sessão. Estudos investigatórios acerca do padrão de relação poderão fornecer mais resultados sobre a adaptabilidade desse instrumento à nossa cultura.

Descritores: Beziehungs-Muster Fragebogen, Relationship Patterns Questionnaire, transferência.

\section{ABSTRACT}

Introduction: The Beziehungs-Muster Fragebogen (BeMus-3), known in the international literature as the Relationship Patterns Questionnaire $(R P Q)$, is a questionnaire that evaluates the central pattern of relationship. The development of its Brazilian Portuguese version aims at introducing, in our country, a self-report measurement to implement research projects involving the construct of transference.

Methodology: The development stages of this version were: obtaining permission from the authors; translation of the original instrument into Brazilian Portuguese; evaluation and adjustment of the translated material by psychiatry and psychology professionals; back-translation; evaluation of semantic equivalence; consensus of psychiatry and psychology professionals on the adequacy of the instrument to our culture; interchange with the target population.

Conclusion: BeMus-3 is a self-report measurement to assess the transference or central pattern of relationship that can facilitate the implementation of projects to investigate the transference-related aspects of therapeutic relationship. This method is easy to apply and analyze, has a low cost and does not require the use of video or voice recorders during the session. Investigative studies on the pattern of relationship may provide further results on the adaptability of this instrument to our culture.

Keywords: Beziehungs-muster fragebogen, relationship patterns questionnaire, transference.

Title: Development of a Brazilian Portuguese version of the questionnaire on relationships pattern Beziehungs-Muster Fragebogen.

Correspondência:

Eliane B. Ferreira

Av. D. Pedro II, 1240/206

CEP 90550-140 - Porto Alegre, RS

Tel.: (51) 9285.7534

E-mail: ebferreira@cpovo.net

Copyright $(\odot$ Revista de Psiquiatria do Rio Grande do Sul - SPRS 


\section{Anexo 1 - Questionário sobre padrão de relacionamentos Beziehungs-Muster Fragebogen}

FORMA ABREVIADA

\begin{tabular}{|l|l|l|l|}
\hline Nome: \\
\hline Sexo: & $\left(\begin{array}{l}\text { ) Masculino } \\
\text { ( ) Feminino }\end{array}\right.$ & Data: & Ano de nascimento: \\
\hline
\end{tabular}

Inicialmente, neste questionário nos interessa a sua relação para com o(a) seu(sua) terapeuta e a sua relação para com uma pessoa muito importante em sua vida, que você vai definir qual é. As questões finais deste questionário deverão ser preenchidas conforme sua relação para com os seus pais, como você os vivenciou na infância.

Favor escrever abaixo o papel da pessoa importante em sua vida - cônjuge, amigo(a), filho(a), etc. - que você escolheu para responder o questionário:

Em resumo, o questionário deverá ser preenchido baseado em:

Sua relação atual com seu(sua) terapeuta;

Sua relação atual (ou num passado recente) com a pessoa importante;

Sua relação com a mãe e o pai na sua infância, até onde você consegue lembrar.

Trabalhe frase por frase e assinale, com relação às pessoas acima mencionadas, o grau de concordância que representa mais a sua situação.

Favor responder, sempre, conforme indicado, todos os itens e assinalar apenas um $X$ em cada linha referente à sua relação com o terapeuta e apenas um $\mathrm{X}$ em cada linha referente à sua relação com a pessoa importante que você escolheu. Muito obrigado!

Exemplo:

\begin{tabular}{|c|c|c|c|}
\hline Comportamento dele/dela & Minha própria reação & Terapeuta & Pessoa importante \\
\hline $\begin{array}{l}\text { Quando ele/ela } \\
\text { age da seguinte forma... }\end{array}$ & Eu faço o seguinte.. & 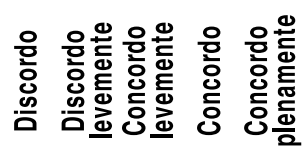 & 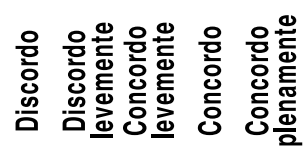 \\
\hline Ele/ela se impõe & $\begin{array}{l}\text { Eu também me imponho } \\
\text { Eu o/a trato amigavelmente } \\
\text { Eu me submeto } \\
\text { Eu o/a agrido verbal ou fisicamente }\end{array}$ & $\begin{array}{l}\square \square \square \square \square \\
\square \square \square \square \square \\
\square \square \square \square \\
\square \square \square \square \square\end{array}$ & $\begin{array}{l}\square \square \square \square \square \\
\square \square \square \square \square \\
\square \square \square \square \\
\square \square \square \square \square\end{array}$ \\
\hline
\end{tabular}


Favor responder, sempre, conforme indicado, todos os itens e assinalar apenas um $\mathrm{X}$ em cada linha referente à sua relação com o terapeuta e apenas um $\mathrm{X}$ em cada linha referente à sua relação com a pessoa importante. Muito obrigado!

1) No geral, qual o seu comportamento em relação ao seu terapeuta e à pessoa importante em sua vida?

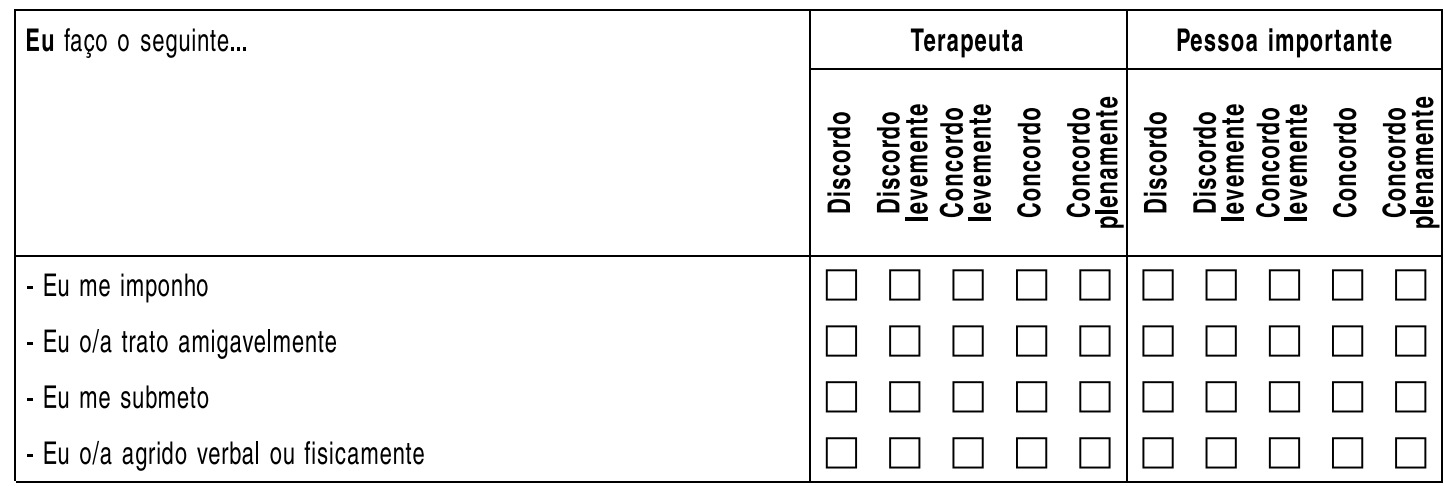

2) No geral, qual o comportamento de seu terapeuta e da pessoa importante em sua vida?

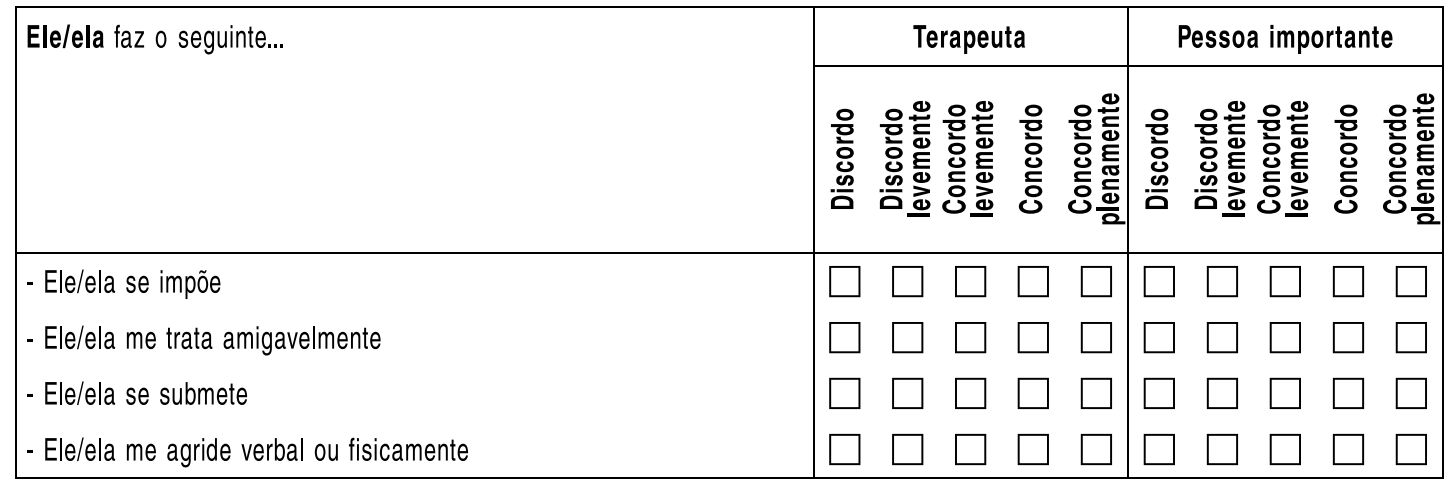

3) O que você sente após um confronto com o seu terapeuta e com a pessoa importante em sua vida?

\begin{tabular}{|c|c|c|c|c|c|}
\hline \multirow{2}{*}{$\begin{array}{l}\text { Após um confronto com ele/ela, } \\
\text { eu me vivencio da seguinte forma... }\end{array}$} & \multicolumn{2}{|c|}{ Terapeuta } & \multicolumn{3}{|c|}{ Pessoa importante } \\
\hline & 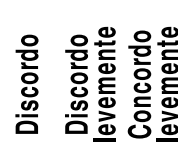 & 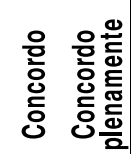 & 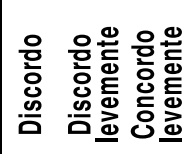 & 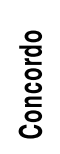 & 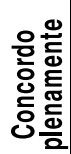 \\
\hline - Eu ajo de forma independente & $\square \square \square$ & $\square \square$ & $\square \square \square$ & $\square$ & $\square$ \\
\hline - Eu sigo minhas próprias idéias & $\square \square \square$ & $\square \square$ & $\square \square \square$ & $\square$ & $\square$ \\
\hline - Eu me trato amigavelmente & $\square \square \square$ & $\square \square$ & $\square \square \square$ & $\square$ & $\square$ \\
\hline - Eu procuro satisfazer as minhas necessidades & $\square \square \square$ & $\square \square$ & $\square \square \square$ & $\square$ & $\square$ \\
\hline - Eu me sujeito às minhas obrigações & $\square \square \square$ & $\square \square$ & $\square \square \square$ & $\square$ & $\square$ \\
\hline - Eu me contenho & $\square \square \square$ & $\square \square$ & $\square \square \square$ & $\square$ & $\square$ \\
\hline - Eu fico bravo comigo mesmo(a) & $\square \square \square$ & $\square \square$ & $\square \square \square$ & $\square$ & $\square$ \\
\hline - Eu descuido das minhas necessidades & $\square \square \square$ & $\square \square$ & $\square \square \square$ & $\square$ & $\square$ \\
\hline
\end{tabular}


BeMus-3 em português do Brasil - Ferreira et al.

4) Como você reage ao comportamento de seu(sua) terapeuta e da pessoa importante em sua vida quando ele/ela se comporta ou caso ele/ela se comportasse da seguinte forma:

\begin{tabular}{|c|c|c|c|}
\hline Comportamento dele/dela & Minha própria reação & Terapeuta & Pessoa importante \\
\hline $\begin{array}{l}\text { Quando ele/ela } \\
\text { age da seguinte forma... }\end{array}$ & Eu faço o seguinte... & 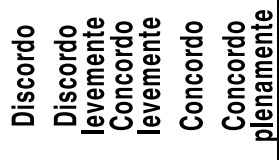 & 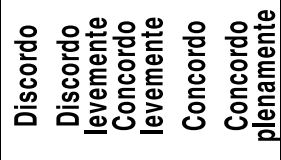 \\
\hline Ele/ela & $\begin{array}{l}\text { Eu também me imponho } \\
\text { Eu o/a trato amigavelmente } \\
\text { Eu me submeto } \\
\text { Eu o/a agrido verbal ou fisicamente }\end{array}$ & $\begin{array}{l}\square \square \square \square \square \\
\square \square \square \square \square \\
\square \square \square \square \square \\
\square \square \square \square \square\end{array}$ & $\begin{array}{l}\square \square \square \square \square \\
\square \square \square \square \square \\
\square \square \square \square \square \\
\square \square \square \square \square\end{array}$ \\
\hline $\begin{array}{l}\text { Ele/ela me trata } \\
\text { amigavelmente }\end{array}$ & $\begin{array}{l}\text { Eu me imponho } \\
\text { Eu também o/a trato amigavelmente } \\
\text { Eu me submeto } \\
\text { Eu o/a agrido verbal ou fisicamente }\end{array}$ & $\begin{array}{l}\square \square \square \square \square \\
\square \square \square \square \square \\
\square \square \square \square \square \\
\square \square \square \square \square\end{array}$ & $\begin{array}{l}\square \square \square \square \\
\square \square \square \square \\
\square \square \square \square \\
\square \square \square \square\end{array}$ \\
\hline Ele/ela se submete & $\begin{array}{l}\text { Eu me imponho } \\
\text { Eu o/a trato amigavelmente } \\
\text { Eu também me submeto } \\
\text { Eu o/a agrido verbal ou fisicamente }\end{array}$ & $\begin{array}{l}\square \square \square \square \square \\
\square \square \square \square \square \\
\square \square \square \square \square \\
\square \square \square \square \square\end{array}$ & $\begin{array}{l}\square \square \square \square \\
\square \square \square \square \\
\square \square \square \square \\
\square \square \square \square\end{array}$ \\
\hline $\begin{array}{l}\text { Ele/ela me agride verbal ou } \\
\text { fisicamente }\end{array}$ & $\begin{array}{l}\text { Eu me imponho } \\
\text { Eu o/a trato amigavelmente } \\
\text { Eu me submeto } \\
\text { Eu também o/a agrido verbal ou fisicamente }\end{array}$ & $\begin{array}{l}\square \square \square \square \square \\
\square \square \square \square \square \\
\square \square \square \square \square \\
\square \square \square \square \square\end{array}$ & $\begin{array}{l}\square \square \square \square \square \\
\square \square \square \square \square \\
\square \square \square \square \square \\
\square \square \square \square \square\end{array}$ \\
\hline
\end{tabular}


5) Qual a reação de seu(sua) terapeuta e da pessoa importante em sua vida em relação a você quando você se comporta ou caso você se comportasse da seguinte forma:

\begin{tabular}{|c|c|c|c|}
\hline \multirow{2}{*}{$\begin{array}{l}\text { Quando eu ajo da } \\
\text { seguinte forma... }\end{array}$} & \multirow[t]{2}{*}{ Ele/ela faz o seguinte... } & Terapeuta & Pessoa importante \\
\hline & & 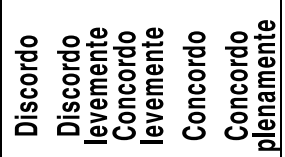 & 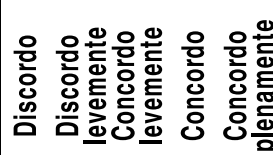 \\
\hline Eu me imponho & $\begin{array}{l}\text { Ele/ela também se impõe } \\
\text { Ele/ela me trata amigavelmente } \\
\text { Ele/ela se submete } \\
\text { Ele/ela me agride verbal ou fisicamente }\end{array}$ & $\begin{array}{l}\square \square \square \square \square \\
\square \square \square \square \square \\
\square \square \square \square \square \\
\square \square \square \square \square\end{array}$ & $\begin{array}{l}\square \square \square \square \square \\
\square \square \square \square \square \\
\square \square \square \square \square \\
\square \square \square \square \square\end{array}$ \\
\hline $\begin{array}{l}\text { Eu o/a trato } \\
\text { amigavelmente }\end{array}$ & $\begin{array}{l}\text { Ele/ela se impõe } \\
\text { Ele/ela também me trata amigavelmente } \\
\text { Ele/ela se submete } \\
\text { Ele/ela me agride verbal ou fisicamente }\end{array}$ & $\begin{array}{l}\square \square \square \square \square \\
\square \square \square \square \square \\
\square \square \square \square \square \\
\square \square \square \square \square\end{array}$ & $\begin{array}{l}\square \square \square \square \square \\
\square \square \square \square \square \\
\square \square \square \square \square \\
\square \square \square \square \square\end{array}$ \\
\hline Eu me submeto & $\begin{array}{l}\text { Ele/ela se impõe } \\
\text { Ele/ela me trata amigavelmente } \\
\text { Ele/ela também se submete } \\
\text { Ele/ela me agride verbal ou fisicamente }\end{array}$ & $\begin{array}{l}\square \square \square \square \square \\
\square \square \square \square \square \\
\square \square \square \square \square \\
\square \square \square \square \square\end{array}$ & $\begin{array}{l}\square \square \square \square \square \\
\square \square \square \square \square \\
\square \square \square \square \square \\
\square \square \square \square \square\end{array}$ \\
\hline $\begin{array}{l}\text { Eu o/a agrido verbal ou } \\
\text { fisicamente }\end{array}$ & $\begin{array}{l}\text { Ele/ela se impõe } \\
\text { Ele/ela também me trata amigavelmente } \\
\text { Ele/ela se submete } \\
\text { Ele/ela também me agride verbal ou fisicamente }\end{array}$ & $\begin{array}{l}\square \square \square \square \square \\
\square \square \square \square \square \\
\square \square \square \square \square \\
\square \square \square \square \square\end{array}$ & $\begin{array}{l}\square \square \square \square \square \\
\square \square \square \square \square \\
\square \square \square \square \square \\
\square \square \square \square \square\end{array}$ \\
\hline
\end{tabular}

Esta parte trata da sua relação com os seus pais quando você era criança.

1) No geral, qual era seu tipo de comportamento em relação ao seu pai e também à sua mãe?

\begin{tabular}{|c|c|c|c|c|}
\hline \multirow[t]{2}{*}{ Eu fazia o seguinte... } & \multicolumn{2}{|l|}{ Pai } & \multicolumn{2}{|c|}{ Mãe } \\
\hline & 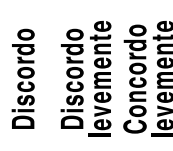 & 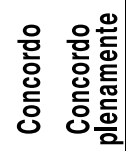 & 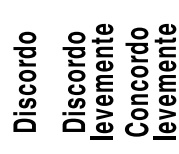 & 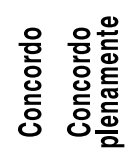 \\
\hline - Eu me impunha & $\square \square \square$ & $\square \square$ & $\square \square \square$ & $\square \square$ \\
\hline - Eu o/a tratava amigavelmente & $\square \square \square$ & $\square \square$ & $\square \square \square$ & $\square \square$ \\
\hline - Eu me submetia & $\square \square \square$ & $\square \square$ & $\square \square \square$ & $\square \square$ \\
\hline - Eu o/a agredia verbal ou fisicamente & $\square \square \square$ & $\square \square$ & $\square \square \square$ & $\square \square$ \\
\hline
\end{tabular}


BeMus-3 em português do Brasil - Ferreira et al.

2) No geral, qual era o comportamento de seu pai e de sua mãe?

\begin{tabular}{|c|c|c|c|c|}
\hline \multirow[t]{2}{*}{ Ele/ela fazia o seguinte... } & \multicolumn{2}{|l|}{ Pai } & \multicolumn{2}{|c|}{ Mãe } \\
\hline & 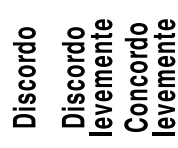 & 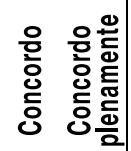 & 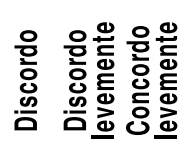 & 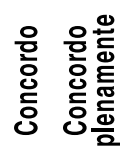 \\
\hline - Ele/ela se impunha & $\square \square \square$ & $\square \square$ & $\square \square \square$ & $\square \square$ \\
\hline - Ele/ela me tratava amigavelmente & $\square \square \square$ & $\square \square$ & $\square \square \square$ & $\square \square$ \\
\hline - Ele/ela se submetia & $\square \square \square$ & $\square \square$ & $\square \square \square$ & $\square \square$ \\
\hline - Ele/ela me agredia verbal ou fisicamente & $\square \square \square$ & $\square \square$ & $\square \square \square$ & $\square \square$ \\
\hline
\end{tabular}

3) Como você se sentia depois de um confronto com seu pai e sua mãe?

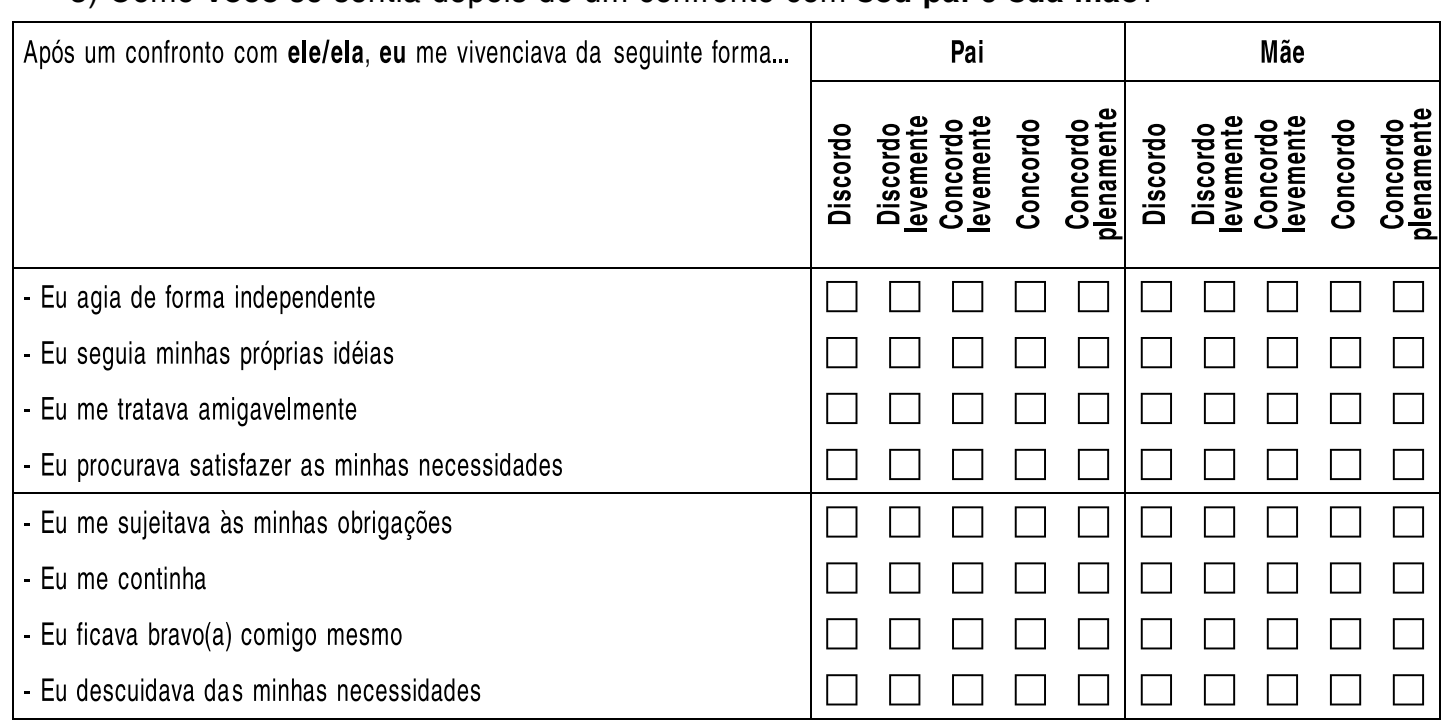


BeMus-3 em português do Brasil - Ferreira et al.

4) Qual era a sua reação, na infância, quando seu pai ou sua mãe se comportavam ou se eles tivessem se comportado da seguinte forma:

\begin{tabular}{|c|c|c|c|}
\hline \multirow{2}{*}{$\begin{array}{l}\text { Quando ele/ela } \\
\text { agia da seguinte forma... }\end{array}$} & \multirow[t]{2}{*}{ Eu fazia o seguinte... } & Pai & Mãe \\
\hline & & 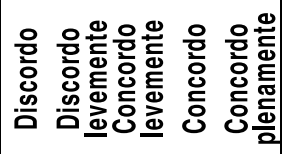 & 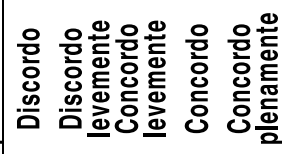 \\
\hline \multirow[t]{4}{*}{ Ele/ela se impunha } & Eu também me impunha & $\square \square \square \square \square$ & $\square \square \square \square \square$ \\
\hline & Eu o/a tratava amigavelmente & $\square \square \square \square \square$ & $\square \square \square \square \square$ \\
\hline & Eu me submetia & $\square \square \square \square \square$ & $\square \square \square \square$ \\
\hline & Eu o/a agredia verbal ou fisicamente & $\square \square \square \square \square$ & $\square \square \square \square$ \\
\hline \multirow{4}{*}{$\begin{array}{l}\text { Ele/ela me tratava } \\
\text { amigavelmente }\end{array}$} & Eu também me impunha & $\square \square \square \square \square$ & $\square \square \square \square$ \\
\hline & Eu também o/a tratava amigavelmente & $\square \square \square \square \square$ & $\square \square \square \square$ \\
\hline & Eu me submetia & $\square \square \square \square \square$ & $\square \square \square \square \square$ \\
\hline & Eu o/a agredia verbal ou fisicamente & $\square \square \square \square \square$ & $\square \square \square \square$ \\
\hline \multirow[t]{4}{*}{ Ele/ela se submetia } & Eu também me impunha & $\square \square \square \square \square$ & $\square \square \square \square$ \\
\hline & Eu o/a tratava amigavelmente & $\square \square \square \square \square$ & $\square \square \square \square$ \\
\hline & Eu também me submetia & $\square \square \square \square \square$ & $\square \square \square \square$ \\
\hline & Eu o/a agredia verbal ou fisicamente & $\square \square \square \square \square$ & $\square \square \square \square \square$ \\
\hline \multirow{4}{*}{$\begin{array}{l}\text { Ele/ela me agredia verbal ou } \\
\text { fisicamente }\end{array}$} & Eu também me impunha & $\square \square \square \square \square$ & $\square \square \square \square$ \\
\hline & Eu o/a tratava amigavelmente & $\square \square \square \square \square$ & $\square \square \square \square$ \\
\hline & Eu me submetia & $\square \square \square \square \square$ & $\square \square \square \square \square$ \\
\hline & Eu também o/a agredia verbal ou fisicamente & $\square \square \square \square \square$ & $\square \square \square \square$ \\
\hline
\end{tabular}


BeMus-3 em português do Brasil - Ferreira et al.

5) Qual era a reação de seu pai e de sua mãe, na infância, em relação a você, quando você se comportava ou caso você se comportasse da seguinte forma:

\begin{tabular}{|c|c|c|c|}
\hline \multirow{2}{*}{$\begin{array}{l}\text { Quando eu agia da } \\
\text { seguinte forma... }\end{array}$} & \multirow[t]{2}{*}{ Ele/ela fazia o seguinte... } & Pai & Mãe \\
\hline & & 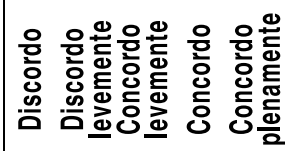 & 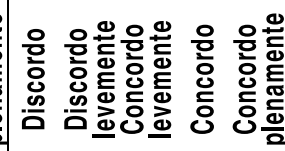 \\
\hline \multirow[t]{4}{*}{ Eu me impunha } & Ele/ela também se impunha & $\square \square \square \square \square$ & $\square \square \square \square$ \\
\hline & Ele/ela me tratava amigavelmente & $\square \square \square \square \square$ & $\square \square \square \square$ \\
\hline & Ele/ela se submetia & $\square \square \square \square \square$ & $\square \square \square \square$ \\
\hline & Ele/ela me agredia verbal ou fisicamente & $\square \square \square \square \square$ & $\square \square \square \square$ \\
\hline \multirow{4}{*}{$\begin{array}{l}\text { Eu o/a tratava } \\
\text { amigavelmente }\end{array}$} & Ele/ela se impunha & $\square \square \square \square \square$ & $\square \square \square$ \\
\hline & Ele/ela também me tratava amigavelmente & $\square \square \square \square \square$ & $\square \square[$ \\
\hline & Ele/ela se submetia & $\square \square \square \square \square$ & $\square \square \square \square$ \\
\hline & Ele/ela me agredia verbal ou fisicamente & $\square \square \square \square \square$ & $\square \square \square \square$ \\
\hline \multirow[t]{4}{*}{ Eu me submetia } & Ele/ela se impunha & $\square \square \square \square \square$ & $\square \square \square \square$ \\
\hline & Ele/ela me tratava amigavelmente & $\square \square \square \square \square$ & $\square \square \square \square \square$ \\
\hline & Ele/ela também se submetia & $\square \square \square \square \square$ & $\square \square \square \square \square$ \\
\hline & Ele/ela me agredia verbal ou fisicamente & $\square \square \square \square \square$ & $\square \square \square \square \square$ \\
\hline \multirow{4}{*}{$\begin{array}{l}\text { Eu o/a agredia verbal } \\
\text { ou fisicamente }\end{array}$} & Ele/ela se impunha & $\square \square \square \square \square$ & $\square \square \square \square$ \\
\hline & Ele/ela me tratava amigavelmente & $\square \square \square \square \square$ & $\square \square \square \square$ \\
\hline & Ele/ela se submetia & $\square \square \square \square \square$ & $\square \square \square \square$ \\
\hline & Ele/ela também me agredia verbal ou fisicamente & $\square \square \square \square \square$ & $\square \square \square \square \square$ \\
\hline
\end{tabular}

Muito obrigado pela sua colaboração! 